\title{
Université d'été : la communication professionnelle en santé La maîtriser, l'enseigner, l'évaluer et la promouvoir ${ }^{\star}$
}

Du 18 au 20 juin 2018 à l'Université de Montréal

\section{Contexte}

Une communication professionnelle efficace est essentielle à la qualité des soins et en est indissociable. Les professionnels de la santé doivent acquérir non seulement un savoir technique et clinique, mais également des habiletés communicationnelles et relationnelles pour faire face à la complexité et à la diversité des tâches qui leur incombent. La maîtrise de la communication en santé ne peut plus être considérée comme périphérique par rapport à l'exercice de la médecine et, de ce fait, doit faire l'objet d'une formation rigoureuse, au même titre que les sciences fondamentales et cliniques.

\section{Public cible}

La formation offerte en français vise tous les individus, quel que soit leur niveau de compétence, impliqués dans l'enseignement de la communication des facultés en santé (médecine, médecine dentaire, pharmacie, sciences infirmières) ou d'écoles spécialisées (orthophonie, ergothérapie, physiothérapie, optométrie, etc.), plus spécifiquement : les enseignants en milieu clinique; les leaders pédagogiques; les directeurs de programme ; les vice-doyens aux études pré et postdoctorales/responsables au niveau des $1^{\mathrm{er}}, 2^{\mathrm{e}}$ et $3^{\mathrm{e}}$ cycles; les chercheurs en pédagogie des sciences de la santé.

\section{Objectifs}

À la fin de l'université d'été, les participants devraient pouvoir :

- maîtriser les bases de la communication professionnelle en santé, incluant l'art de l'entretien clinique adapté à la variété des pratiques ;

\footnotetext{
^ Formation en demande d'accréditation.
}

- choisir et utiliser des méthodes pédagogiques adaptées à leur environnement pour l'apprentissage de la communication professionnelle en santé, incluant l'observation directe et la rétroaction;

- proposer des méthodes élémentaires d'évaluation;

- collaborer à et gérer la mise en place d'un curriculum en communication adapté à leur discipline, leur milieu de pratique et au niveau de formation ciblé.

\section{Pourquoi participer?}

Pour se retrouver durant trois jours (20 à 30 participants) dans un environnement d'apprentissage de la communication professionnelle en santé, dynamique, interactif et inédit dans la francophonie médicale.

Pour s'exercer à améliorer ses compétences communicationnelles, entouré d'experts à la fine pointe des connaissances.

Pour devenir un leader de la compétence «communication » dans son milieu de pratique.

Pour profiter des attraits de Montréal à une période festive de l'année.

Pour faire partie d'un réseau de collègues partageant un intérêt pour la communication professionnelle en santé.

\section{Programme préliminaire}

Jour 1 (8h30-16h30)

Les habiletés communicationnelles

Jour 2 (8h30-16h30)

La supervision des habiletés communicationnelles

Jour 3 (8h30-16h30) 
L'évaluation des habiletés communicationnelles et le leadership en enseignement de la communication

\section{Comité Organisateur}

(tous membres du groupe de travail sur la communication professionnelle de la SIFEM)

Dr. Olivier Jamoulle MD, FRCP (président)

Pre Marie-Thérèse Lussie, MD, MSC, FCMFC

Claude Richard, PhD

Pr. Bernard Millette MD, MSc, FCMCF

Dr Sophie Galarneau MD, MCMFC

Emma Glaser MD (candidate), BSc, MSc
Les informations sur les détails des journées, les inscriptions, les modalités de transport et de logement se retrouvent sur le site web du Centre de pédagogie appliquée aux sciences de la santé (CPASS) https://www.cpass.umontreal.ca.

Le prix d'inscription pour les trois jours est de:

$600 \$$ Can (+taxes) avant le 20 avril 2018

$700 \$$ Can (+taxes) à partir du 21 avril 2018.

Dans la situation d'un nombre insuffisant d'inscriptions en date du 21 avril 2018, le comité planificateur de l'Université d'été 2018 se réserve le droit d'annuler l'événement à cette date. 